\title{
Matter-Wave Tractor Beams
}

\section{Gorlach, Alexey A.; Gorlach, Maxim A.; Lavrinenko, Andrei; Novitsky, Andrey}

\section{Published in:}

Physical Review Letters

Link to article, DOI:

10.1103/PhysRevLett.118.180401

Publication date:

2017

\section{Document Version}

Publisher's PDF, also known as Version of record

Link back to DTU Orbit

\section{Citation (APA):}

Gorlach, A. A., Gorlach, M. A., Lavrinenko, A., \& Novitsky, A. (2017). Matter-Wave Tractor Beams. Physical Review Letters, 118(18), [180401]. https://doi.org/10.1103/PhysRevLett.118.180401

\section{General rights}

Copyright and moral rights for the publications made accessible in the public portal are retained by the authors and/or other copyright owners and it is a condition of accessing publications that users recognise and abide by the legal requirements associated with these rights.

- Users may download and print one copy of any publication from the public portal for the purpose of private study or research.

- You may not further distribute the material or use it for any profit-making activity or commercial gain

- You may freely distribute the URL identifying the publication in the public portal 


\title{
Matter-Wave Tractor Beams
}

\author{
Alexey A. Gorlach, ${ }^{1}$ Maxim A. Gorlach, ${ }^{2}$ Andrei V. Lavrinenko, ${ }^{3}$ and Andrey Novitsky ${ }^{1,3, *}$ \\ ${ }^{1}$ Belarusian State University, 220030 Minsk, Belarus \\ ${ }^{2}$ ITMO University, 197101 St. Petersburg, Russia \\ ${ }^{3}$ Technical University of Denmark, DK-2800 Kongens Lyngby, Denmark
}

(Received 9 January 2017; published 4 May 2017)

\begin{abstract}
Optical and acoustic tractor beams are currently the focus of intense research due to their counterintuitive property of exerting a pulling force on small scattering objects. In this Letter we propose a matter-wave tractor beam and utilize the de Broglie waves of nonrelativistic matter particles in analogy to "classical" tractor beams. We reveal the presence of the quantum-mechanical pulling force for the variety of quantum mechanical potentials observing the resonant enhancement of the pulling effect under the conditions of the suppressed scattering known as the Ramsauer-Townsend effect. We also derive the sufficient conditions on the scattering potential for the emergence of the pulling force and show that, in particular, a Coulomb scatterer is always shoved, while a Yukawa (screened Coulomb) scatterer can be drawn. Pulling forces in optics, acoustics, quantum mechanics, and classical mechanics are compared, and the matter-wave pulling force is found to have exclusive properties of dragging slow particles in short-range potentials. We envisage that the use of tractor beams could lead to the unprecedented precision in manipulation with atomic-scale quantum objects.
\end{abstract}

DOI: 10.1103/PhysRevLett.118.180401

Maxwell's theory of electromagnetism [1] was validated in a series of seminal experiments, one of which demonstrated the existence of the radiation pressure [2]. Since then it has been commonly accepted that nongradient light exerts a pushing force on objects. The situation changed after discovery of an unexpected pulling force in the fields of gradientless structured light (so-called tractor beams) [3-7], widely modeled by nondiffractive Bessel beams. In both optics and acoustics [8], the pulling force originates from the enhanced forward light scattering implying its field momentum increases, while a scatterer gains a backward momentum in concordance with the momentum conservation. This idea has been widely exploited to reverse the force direction due to the environment. Examples include the interface between two media [9-11], plasmonic interfaces [12,13], and anisotropic materials [14-16]. The pulling force also depends on the material of the scattering object and can be accordingly observed for gain [17], chiral $[18,19]$, and structured [20] particles. Experiments with tractor beams $[21,22]$ confirm their importance for optical micromanipulation $[23,24]$. The pulling force has been also studied with classical electromagnetic fields interacting with atoms $[25,26]$.

In analogy with the tractor beams in optics and acoustics, we propose the concept of the matter-wave pulling forces in quantum mechanics. The matter-wave tractor beams are different from optical and acoustic ones, because (i) the incident beam is a beam of particles (e.g., electrons) or, equivalently, a wave packet according to the wave-particle duality, and (ii) the forces have a quantum probabilistic nature. Remarkably, nongradient matter-wave packets have been demonstrated recently: generation of both Airy and Bessel wave packets of electrons using the technique of nanoscale holograms is reported in Refs. [27,28].

The stationary wave function

$$
\psi_{E, \alpha}(\mathbf{r})=A_{0} \exp \left(i k_{0} \cos \alpha z\right) J_{0}\left(k_{0} \sin \alpha \rho\right)
$$

in the form of the zeroth-order Bessel function $J_{0}(\xi)$ is a solution of the stationary Schrödinger equation for nonrelativistic particles of mass $m$ and energy $E$ propagating in the $z$ direction, where $\mathbf{r}=(x, y, z)$ is the radius vector, $\rho=\sqrt{x^{2}+y^{2}}, k_{0}=\sqrt{2 m E} / \hbar$ is the wave number, $k_{0} \cos \alpha$ and $k_{0} \sin \alpha$ are the longitudinal and transverse wave numbers, respectively, and $\hbar$ is the reduced Planck constant. The Bessel wave function is a superposition of plane wave functions for which wave vectors are located on a cone of apex angle $2 \alpha$ [see Fig. 1(a)]. Since $\partial\left|\psi_{E, \alpha}\right|^{2} / \partial z=0$, the Bessel wave function is gradientless along the $z$ axis. The envelope, $g$, of the quasistationary Bessel wave packet

$$
\Psi(\mathbf{r}, t)=\psi_{E, \alpha}(\mathbf{r}) e^{-i E t / \hbar} g\left(t-\frac{z}{v_{g r}}\right)
$$

moves with the group velocity $v_{g r}=\sqrt{2 E / m}(\cos \alpha)^{-1}$. For details we refer to Secs. S3 and S4 in Ref. [29].

Interaction of the incident wave packet with a scattering particle positioned at $\mathbf{r}=0$ is described with the help of a spherically symmetric potential energy $U(r)$. Particles in the incident beam are affected by the quantum average force $\left\langle\mathbf{F}_{i}\right\rangle=\langle-\nabla U\rangle$, while the force exerted on the scatterer is 
(a)

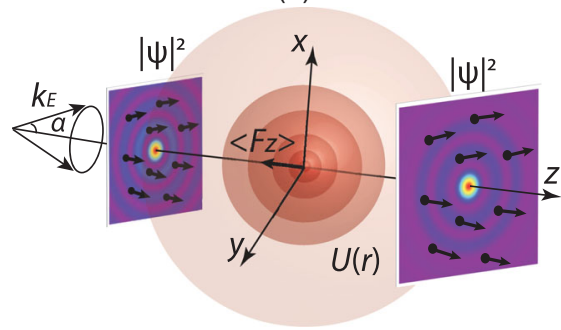

(b)

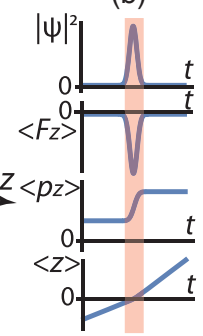

FIG. 1. Wave packet scattering. (a) Sketch of interaction of the beam of particles (Bessel wave function) with a scatterer described by a potential energy $U(r)$. (b) Time dynamics of $\left|\Psi_{t}(0, t)\right|^{2}$, force exerting on the scatterer $\left\langle F_{z}\right\rangle(t)$, momentum $\left\langle p_{z}\right\rangle(t)$, and coordinate $\langle z\rangle(t)$ of the incident quasistationary wave packet (2). Here $g(t)=C \exp \left(-\sigma^{2} t^{2} / 2 \hbar^{2}\right)$, where $C$ is a constant and $\sigma \ll E_{0}$ (see Sec. S5 in [29]). When the wave packet arrives at the scattering center (the peak in the uppermost plot), the force acting on the scatterer arises (pulling force $F_{z}<0$ in the second plot). The incident particle's momentum change $\Delta p_{z}=\left|F_{z}\right| \sqrt{\pi} \hbar / \sigma>0$ is shown in the third plot. The dependence of the coordinate on time is shown in the bottom plot.

$$
\langle\mathbf{F}\rangle=-\left\langle\mathbf{F}_{i}\right\rangle=\langle\nabla U\rangle=\int \Psi_{t}^{*} \nabla U \Psi_{t} d V=\mathbf{F}|g(t)|^{2},
$$

where the latter equality follows from the assumption that the interaction between the incident wave packet and scatterer mainly occurs in the vicinity of the scatterer $\left[g\left(t-z / v_{g r}\right) \approx g(t)\right] . \Psi_{t}$ is the total wave packet describing the superposition of the incident and scattered waves, and $|\mathbf{F}|=F_{z}$ is the $z$-oriented stationary force. The Ehrenfest theorem yields the averaged momentum acquired by the incident particle due to the interaction with the scatterer as $\Delta p_{z}=-F_{z} \int_{-\infty}^{\infty}|g(t)|^{2} d t$. Provided the force acting on the scatterer is negative, the particles of the incident beam increase momentum, providing evidence of the quantum pulling force (see Sec. S16 in Ref. [29]). Time evolution of some averaged quantities is demonstrated in Fig. 1(b).

Hereinafter we inspect the stationary force $F_{z}$ and reveal the conditions for the force to be backward. Using the stress-tensor technique conventionally employed in electrodynamics and extended to quantum mechanics, e.g., in Refs. [34-36], we write the stationary force $\mathbf{F}=-\oint \stackrel{\leftrightarrow}{T} \cdot d \mathbf{s}$, where the integration surface should be chosen at infinity. As shown in [29] (Sec. S6), the stress tensor $\stackrel{\leftrightarrow}{T}=$ $\left(\hbar^{2} / 2 \mu\right)\left(\nabla \psi_{t} \otimes \nabla \psi_{t}^{*}+\nabla \psi_{t}^{*} \otimes \nabla \psi_{t}\right)-\overleftrightarrow{I}\left[(U-E)\left|\psi_{t}\right|^{2}+\right.$ $\left.\left(\hbar^{2} / 2 \mu\right)\left|\nabla \psi_{t}\right|^{2}\right]$ is expressed by means of potential energy $U(r)$ and total stationary wave function $\psi_{t}(\mathbf{r})=$ $R_{l}(r) Y_{l m}(\theta, \varphi) / k_{0} r$, where $r, \theta$, and $\varphi$ are the spherical coordinates, $R_{l}(r)$ is the radial wave function, $Y_{l m}(\theta, \varphi)$ is the spherical harmonic, $\mu$ is the reduced mass, $\otimes$ stands for the tensor product, and $\overleftrightarrow{I}$ is the identity tensor. Radial wave function $R_{l}$ is the solution of the radial Schrödinger equation. Since the integration surface is at infinity, the farfield total wave function $\psi_{t}=\psi_{i}+f(\theta, \varphi) \exp \left(i k_{0} r\right) / r$ is required to carry out the force calculations, where $\psi_{i}$ is the wave function of the incident particle beam and $f(\theta, \varphi)$ is the scattering amplitude.

Adopting the well-established method of phase shifts [37-40], the stationary force for the incident Bessel wave function (1) reads (see Sec. S8 in [29])

$$
F_{z}=\sum_{l=0}^{\infty} F_{l}(E, U) P_{l+1}(\cos \alpha) P_{l}(\cos \alpha),
$$

where $F_{l}(E, U)=F_{N}(l+1) \sin ^{2}\left(\delta_{l+1}-\delta_{l}\right) \geq 0, \quad F_{N}=$ $4 \pi \hbar^{2}\left|A_{0}\right|^{2} / \mu$, and $P_{l}$ is the Legendre polynomial. Phase shifts $\delta_{l}(E, U)$ are defined according to $\tan \delta_{l}=$ $-\left(2 \mu / \hbar^{2}\right) \int_{0}^{\infty} U(r) R_{l}(r) j_{l}\left(k_{0} r\right) r d r$, where $k_{0}=\sqrt{2 \mu E} / \hbar$, $j_{l}$ is the spherical Bessel function of order $l$, and the radial function asymptotic behaves at infinity as $R_{l}(r \rightarrow \infty)=$ $\sin \left(k_{0} r-\pi l / 2\right)+\tan \delta_{l} \cos \left(k_{0} r-\pi l / 2\right)$.

Since $F_{l} \geq 0$, the backward force can be realized only due to the negative Legendre polynomials. Since the term $l=0$ in Eq. (4) is positive, the pulling force $F_{z}<0$ appears owing to the terms with $l \geq 1$. When one keeps the first two terms $(l \leq 1)$ in series (4), condition $\alpha>\arccos (1 / \sqrt{3}) \approx 55^{\circ}$ holds true. If the first three terms are left $(l \leq 2)$, the range of cone angles reads $39^{\circ}<\alpha<55^{\circ}$. The matter-wave pulling force may be enhanced by canceling the positive term $l=0$ in Eq. (4). The equation $F_{0}(E, U)=0$ is exactly the condition for resonant transparency of quantum barriers known in quantum scattering theory as the RamsauerTownsend effect $[41,42]$. The canceling of $F_{l}(E, U)=0$ requires $\delta_{l+1}-\delta_{l}=\pi n_{l}$, where $n_{l}$ is an integer number.

First, we consider a short-range potential using the model of spherical potential barrier $U(r)=U_{0} H(R-r)$ with the radius of interaction $R$, where $H(x)$ is the Heaviside step function. As shown in [29] (Sec. S10), conditions $F_{0}=0$ and $F_{0}=F_{1}=0$ are fulfilled when $j_{0}(k R)=0$ and $j_{1}(k R)=0$, respectively, where $k=$ $\sqrt{2 \mu\left(E-U_{0}\right)} / \hbar$. The backward force stems from the Ramsauer-Townsend effect at low energies $E$. Thus, we can expect that the pulling effect in quantum mechanics is often accompanied by the suppressed scattering cross section and, as a consequence, the pulling force is smaller than the normal, pushing one. The backward force exists both for slow and fast incident particles, as demonstrated in Fig. 2 and Fig. S2 in [29], the wave number $k$ being real (the waves at $r \leq R$ are not evanescent).

According to Fig. 2(a), the quantum pulling force exists in the Born approximation $\left(|U| \ll E_{0}\right)$ [37,38]. Importantly, there is a vast family of potentials beyond the step-function potential focusing the wave function in the forward direction and, thus, ensuring the negative transferred momentum. Applying the sufficient condition $U^{\prime}(0) \int_{0}^{\infty} U(r) r^{2} d r>0$ of the negative matter-wave force in the Born approximation (see derivation in Sec. S12 of 


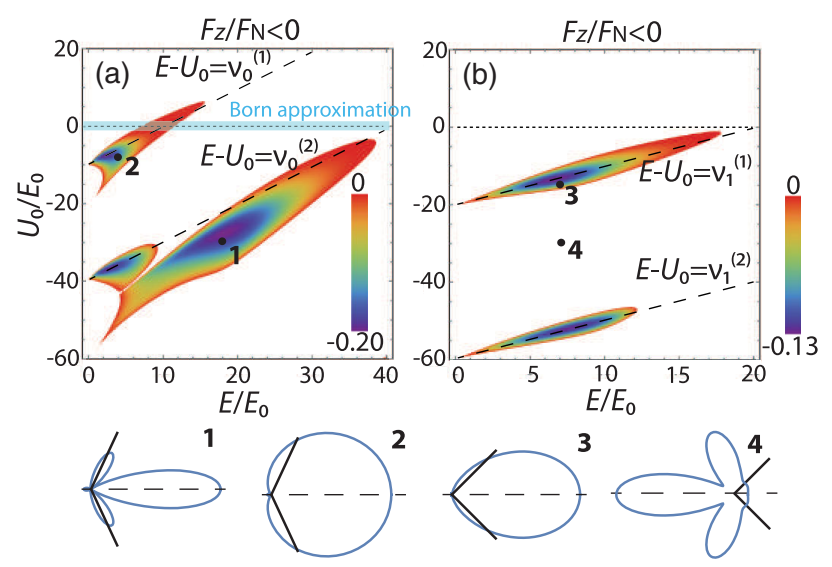

FIG. 2. Pulling-force diagrams $F_{z}\left(E, U_{0}\right)<0$ for a short-range potential modeled as a spherical potential barrier. The dashed lines $E-U_{0}=\nu_{l}^{i}$, where $\nu_{l}^{i}$ is the $i$ th solution of the equation $j_{l}(k R)=0$, indicate (a) $F_{0}=0$ for cone angle $\alpha=65^{\circ}$ and (b) $F_{0}=F_{1}=0$ for $\alpha=45^{\circ}$. The differential cross section $|f(\theta)|^{2}$ at points $1-3$ clearly shows the enhancement of forward scattering (cones with half apex angles $\alpha=65^{\circ}$ and $\alpha=45^{\circ}$ are shown with the black solid lines). Energy normalization parameter $E_{0}=\hbar^{2} / 2 \mu R^{2}$.

[29]) to the Morse potential shown in Fig. 3(a), we arrive at the constraint on the equilibrium position $0<b / R<4 \ln 2 \approx 2.77$. The calculated negative force for cone angle $\alpha=70^{\circ}$ is in the range $1.25<b / R<2.77$ and does fall into the predicted interval of the equilibrium positions [see the diagram $F_{z}(b, E)<0$ in Fig. 3(b)].

The long-range asymptotic behavior of the potential can be modeled assuming the short-range core $U_{0}=$ const for $r \leq R$ and the long-range tail $U_{0}(r / R)^{-n}$ for $r>R$, as rendered in the inset in Fig. 3(c). For small $n<4$ we observe the degradation of the backward force. The pulling force at low cone angles fades away, but the low-energy backward force is still present, as shown for $n=1$ in Fig. 3(c). Diagrams for $n$ from 1 to 6 are shown in Fig. S4 in [29]. The case $n=4$ may be employed in the elastic electron scattering by an atom, with the value of $U_{0}$ being proportional to the dipole polarizability [43].

Finally, we consider potentials without the short-range core. The problem of scattering by the Coulomb potential $U(r)=\gamma / r$ has a well-known solution [37]. The coefficients $F_{l}=F_{N} \kappa^{2}(l+1) /\left[(l+1)^{2}+\kappa^{2}\right]$ (here $\left.\kappa=\mu \gamma / k_{0} \hbar^{2}\right)$ introduced into Eq. (4) ensure that the force is positive [see Fig. 3(d)]. However, when the potential decays faster, the situation may change. The Yukawa or screened Coulomb potential [see the inset in Fig. 3(c)] has exponential asymptotic dependence and, effectively, can be treated as a shortrange potential barrier of some effective height $U_{0}$. Despite the large cone angle $\alpha=85^{\circ}$ the evidence of the matter-wave pulling force for slow incident particles is clear [see Fig. 3(e)]. The details of the numerical calculations carried out for the Yukawa potential can be found in Sec. S14 of [29].

It is instructive to compare the conditions of nascence of the backward forces for optical, acoustic, classical mechani$\mathrm{cal}$, and matter-wave tractor beams. The common feature of the negative forces, the forward scattering enhancement, occurs in quantum mechanics too (see Fig. 2) and, thus, can be treated as a fingerprint of the pulling force. The pulling force in optics originates from the interaction of electric and magnetic dipole moments for $\alpha>60^{\circ}$ and higher-order multipoles otherwise [see Fig. 4(a)]. Interestingly, there is a pronounced difference between electrodynamics or acoustics and quantum mechanics related to the low-energy limit. We cannot obtain the low-energy dragging forces for optical energies approaching zero (Rayleigh approximation), if the
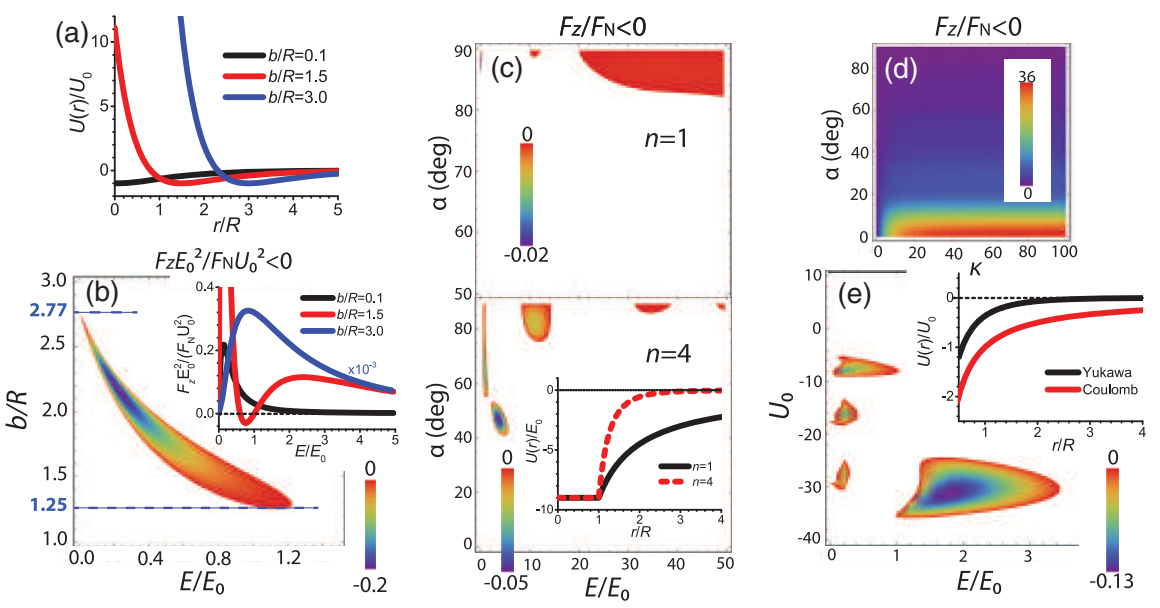

FIG. 3. Matter-wave pulling forces $F_{z}<0$ for nonstep potentials $U(r)$. (a) Function of the Morse potential $U(r)=$ $U_{0}\{\exp [-2(r-b) / R]-2 \exp [-(r-b) / R]\}$ for different equilibrium positions $b$. (b) Backward force for the Morse potentials within the Born approximation $\left(\alpha=70^{\circ}\right)$. Several curves of the force versus energy $E$ are depicted in the inset. (c) Dependence of the force on the asymptotic behavior of the potential $U(r \rightarrow \infty)=U_{0}(r / R)^{-n}$. The cases $n=1$ and $n=4$ are highlighted here (the shape of the potential is shown in the inset). (e) Because of its rapid decay, the Yukawa potential $U(r)=U_{0}(r / R)^{-1} \exp (-r / R)$ possesses pulling properties $\left(\alpha=85^{\circ}\right.$ ), while (d) the Coulomb potential $U(r)=U_{0}(r / R)^{-1}$ does not. In the inset of (e), the Yukawa potential against the Coulomb potential is shown. 
(a) Electrodynamics
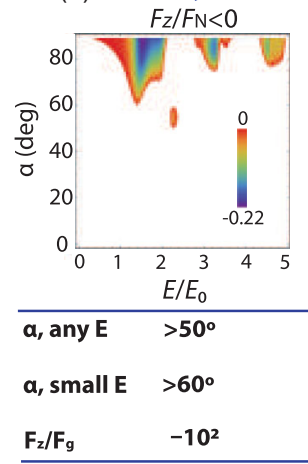

(b) Classical mechanics
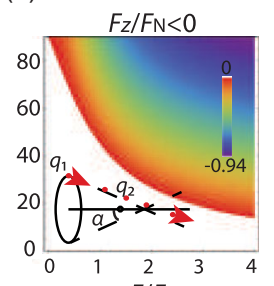

(c) Acoustics

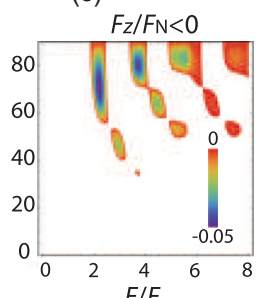

(d) Quantum mechanics

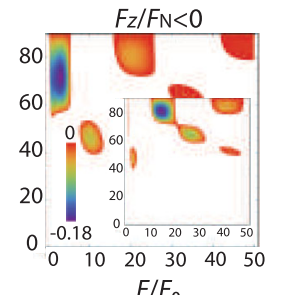

near $90^{\circ}$

-

$>39^{\circ}$

$>39^{\circ}$

$-10^{2}$

FIG. 4. (a) Optical or electrodynamic, (b) classical, (c) acoustic, and (d) matter-wave tractor beams exhibiting the pulling forces $F_{z}(E, \alpha)<0$. (a) Dielectric particles of radius $R$ cannot be pulled by an optical tractor beam [3,4] of low photon energy $E=\hbar \omega$ (silica bead permittivity $\varepsilon=3.9$ and permeability $\mu=1$ and Bessel beam order $m=1$ ). Here $E_{0}=\hbar c / R$ and $F_{N}=c^{2}|A|^{2} / \omega^{2}$, where $c$ is the speed of light, $\omega$ is the circular frequency, and $A$ is the Bessel beam amplitude. (b) Pulling force in classical mechanics is demonstrated as the result of Coulomb scattering to the interior of the cone, as shown in the inset. Normalization coefficients $E_{0}=\left|q_{1} q_{2}\right| / 2 \rho_{0}$ and $F_{N}=4 n_{1} E_{0}$, where $q_{1}$ and $q_{2}$ are charges, $\rho_{0}$ is the impact parameter, and $n_{1}$ is the linear particle concentration. (c) Backward force exerted by the acoustic tractor beam [8] emerges for cone angles below $39^{\circ}$ as shown for carbon tetrachloride sphere of radius $R$ in water ( $\sigma=0.619$ and $\lambda=1.587$ ). We use $E_{0}=\hbar c_{0} / R$ and $F_{N}=\pi R^{2} I_{0} / c_{0} \cos \alpha$, where $c_{0}$ is the speed of sound in the ambient liquid and $I_{0}$ is the acoustic intensity. (d) For matter-wave tractor beams, pulling force below $39^{\circ}$ is impossible, but the backward force may emerge for small energies $E$ in the wide range of cone angles $\alpha>39^{\circ} . U_{0} / E_{0}=-9$ and $U_{0} / E_{0}=-17.5$ in the figure and inset, respectively.

scatterers are nonmagnetic. Therefore, the case of quantum mechanics should be treated equivalent to the magnetodielectric scenario in optics (see Sec. S11 in [29]). The dragging forces for $\alpha<39^{\circ}$ in acoustics [Fig. 4(c)] can be explained similarly to the quantum mechanics case with two-parametric potentials $U\left(r, p_{1}, p_{2}\right)$ through simultaneously satisfying $F_{0}\left(E, p_{1}, p_{2}\right)=F_{1}\left(E, p_{1}, p_{2}\right)=F_{2}\left(E, p_{1}, p_{2}\right)=0$ in Eq. (4). There are precisely two parameters in acoustics, inner-to-outer fluid sound speed $\sigma$ and density $\lambda$ ratios. Classical mechanics stands apart for two reasons: First, the particles cannot be treated as waves. Second, the beam of particles is not nondiffractive in the sense of keeping crosssectional distribution of particles invariant during propagation (however, all particles have the same projection of the longitudinal momentum). Nevertheless, we consider a classic mechanics analogue of a wave tractor beam sketched in the inset of Fig. 4(b). Charged particles are volleyed out from the ring and move toward the scatterer along the generatrix of the cone with apex angle $2 \alpha$. The particles should fall into the cone after the Coulomb scattering to increase the forward momentum and result in the backward force (see Sec. S2 in [29]). The matter-wave pulling forces are similar to those in acoustics except that there are no ways to pull back the scatterer with cone angles below $39^{\circ}$ with a single-parametric step potential. In contrast to the pulling forces in electrodynamics, acoustics, and classical mechanics, quantum mechanics offers a low-energy pulling effect in the extraordinarily wide range of cone angles $\alpha>39^{\circ}$ in the vicinity of the Ramsauer-Townsend values [see Fig. 4(d)]. Quantitative estimations of the pulling forces in optics, acoustics, and quantum mechanics normalized to the gravity force of the scatterer are shown in the lowest line of the table in Fig. 4. This normalized quantum pulling force is of the order of the optical pulling force, holding out the hope that our predictions can be confirmed in experiments with cold atoms and molecules. The details of the calculations are provided in Sec. S17 of Ref. [29].

To summarize, the matter-wave tractor beams can play an important part in future applications. De Broglie wavelengths $\lambda_{d B} \sim 10^{-11} \mathrm{~m}$ of nonrelativistic particles bring the hope of achieving an extremely high resolution unattainable by optical means. Another aspect of the small wavelengths is feasible manipulation with quantum particles on the atomic scale. The very existence of the matter-wave pulling force is an issue of fundamental importance. Amazingly, completely different probabilistic interpretations of quantum mechanics do not harm the pulling force phenomenon, thus confirming the wave nature as the uniting principle of all previous studies [3-6,8]. Matterwave tractor beams exhibit pulling forces in the vicinity of the Ramsauer-Townsend energies and attract scatterers with long-range potentials. The quantum mechanical predictions regarding the negative force can be verified either directly (see the discussion of a possible experimental setup in Sec. S16 of Ref. [29]) or using the optical analogy. In the latter case, the short-range potential energy could be realized as a frequency-dispersive spherical bead embedded into a homogeneous medium as outlined in Sec. 15 of [29].

The authors acknowledge financial support from the Belarusian Republican Foundation for Fundamental Research (Grant No. F16R-049) and partial financial support from the Villum Fonden via the DarkSILD project. The authors thank J. Iles-Smith and S. S. Chuchurka for valuable discussions. 
${ }^{*}$ Corresponding author anov@ fotonik.dtu.dk

[1] J.C. Maxwell, A Treatise on Electricity and Magnetism (Dover Publications, New York, 1954).

[2] P. Lebedew, Untersuchungen über die druckkräfte des lichtes, Ann. Phys. (Berlin) 311, 433 (1901).

[3] J. Chen, J. Ng, Z. Lin, and C. T. Chan, Optical pulling force, Nat. Photonics 5, 531 (2011).

[4] A. Novitsky, C.-W. Qiu, and H. Wang, Single Gradientless Light Beam Drags Particles as Tractor Beams, Phys. Rev. Lett. 107, 203601 (2011).

[5] S. Sukhov and A. Dogariu, Negative Nonconservative Forces: Optical "Tractor Beams" for Arbitrary Objects, Phys. Rev. Lett. 107, 203602 (2011).

[6] D. B. Ruffner and D. G. Grier, Optical Conveyors: A Class of Active Tractor Beams, Phys. Rev. Lett. 109, 163903 (2012).

[7] A. Dogariu, S. Sukhov, and J. J. Sáenz, Optically induced 'negative forces,' Nat. Photonics 7, 24 (2013).

[8] P. L. Marston, Axial radiation force of a Bessel beam on a sphere and direction reversal of the force, J. Acoust. Soc. Am. 120, 3518 (2006).

[9] V. Kajorndejnukul, W. Ding, S. Sukhov, C.-W. Qiu, and A. Dogariu, Linear momentum increase and negative optical forces at dielectric interface, Nat. Photonics 7, 787 (2013).

[10] M. Mansuripur, Momentum exchange effect, Nat. Photonics 7, 765 (2013).

[11] C.-W. Qiu, W. Ding, M. R. C. Mahdy, D. Gao, T. Zhang, F. C. Cheong, A. Dogariu, Z. Wang, and C. T. Lim, Photon momentum transfer in inhomogeneous dielectric mixtures and induced tractor beams, Light Sci. Appl. 4, e278 (2015).

[12] A. V. Maslov, Resonant Pulling of a Microparticle Using a Backward Surface Wave, Phys. Rev. Lett. 112, 113903 (2014).

[13] M. I. Petrov, S. V. Sukhov, A. A. Bogdanov, A. S. Shalin, and A. Dogariu, Surface plasmon polariton assisted optical pulling force, Laser Photonics Rev. 10, 116 (2016).

[14] A. Salandrino and D. N. Christodoulides, Reverse optical forces in negative index dielectric waveguide arrays, Opt. Lett. 36, 3103 (2011).

[15] J. Nemirovsky, M. C. Rechtsman, and M. Segev, Negative radiation pressure and negative effective refractive index via dielectric birefringence, Opt. Express 20, 8907 (2012).

[16] A. S. Shalin, S. V. Sukhov, A. A. Bogdanov, P. A. Belov, and P. Ginzburg, Optical pulling forces in hyperbolic metamaterials, Phys. Rev. A 91, 063830 (2015).

[17] A. Mizrahi and Y. Fainman, Negative radiation pressure on gain medium structures, Opt. Lett. 35, 3405 (2010).

[18] K. Ding, J. Ng, L. Zhou, and C. T. Chan, Realization of optical pulling forces using chirality, Phys. Rev. A 89, 063825 (2014).

[19] A. Canaguier-Durand and C. Genet, Chiral route to pulling optical forces and left-handed optical torques, Phys. Rev. A 92, 043823 (2015).

[20] A. Novitsky and C.-W. Qiu, Pulling extremely anisotropic lossy particles using light without intensity gradient, Phys. Rev. A 90, 053815 (2014).

[21] O. Brzobohatý, V. Karásek, M. Šiler, L. Chvátal, T. Čižmár, and P. Zemánek, Experimental demonstration of optical transport, sorting and self-arrangement using a "tractor beam,' Nat. Photonics 7, 123 (2013).

[22] A. Marzo, S. A. Seah, B. W. Drinkwater, D. R. Sahoo, B. Long, and S. Subramanian, Holographic acoustic elements for manipulation of levitated objects, Nat. Commun. 6, 8661 (2015).

[23] A. Ashkin, J. M. Dziedzic, J. E. Bjorkholm, and S. Chu, Observation of a single-beam gradient force optical trap for dielectric particles, Opt. Lett. 11, 288 (1986).

[24] D. G. Grier, A revolution in optical manipulation, Nature (London) 424, 810 (2003).

[25] I. V. Krasnov, Bichromatic optical tractor beam for resonant atoms, Phys. Lett. A 376, 2743 (2012).

[26] M. Sadgrove, S. Wimberger, and S. N. Chormaic, Quantum coherent tractor beam effect for atoms trapped near a nanowaveguide, Sci. Rep. 6, 28905 (2016).

[27] N. Voloch-Bloch, Y. Lereah, Y. Lilach, A. Gover, and A. Arie, Generation of electron Airy beams, Nature (London) 494, 331 (2013).

[28] V. Grillo, E. Karimi, G. C. Gazzadi, S. Frabboni, M. R. Dennis, and R.W. Boyd, Generation of Nondiffracting Electron Bessel Beams, Phys. Rev. X 4, 011013 (2014).

[29] See Supplemental Material at http://link.aps.org/ supplemental/10.1103/PhysRevLett.118.180401 for detailed analytical derivations and discussion of Figs. 2-4, which includes Refs. [30-33].

[30] L. D. Landau and E. M. Lifshitz, Mechanics (ButterworthHeinemann, Oxford, 1976).

[31] M. V. Berry and N. L. Balazs, Nonspreading wave packets, Am. J. Phys. 47, 264 (1979).

[32] S. Grimme, Accurate description of van der Waals complexes by density functional theory including empirical corrections, J. Comput. Chem. 25, 1463 (2004).

[33] E. O. Gadamer, UTIA Report No. 83, 1962.

[34] O. H. Nielsen and R.M. Martin, Quantum-mechanical theory of stress and force, Phys. Rev. B 32, 3780 (1985).

[35] B. M. Deb and S. K. Ghosh, On some 'local' force densities and stress tensors in molecular quantum mechanics, J. Phys. B 12, 3857 (1979).

[36] R.E. Wyatt, Quantum Dynamics with Trajectories: Introduction to Quantum Hydrodynamics (Springer-Verlag, New York, 2005).

[37] L. D. Landau and E. M. Lifshitz, Quantum Mechanics: Non-Relativistic Theory (Pergamon Press, Oxford, 1977).

[38] A. Messiah, Quantum Mechanics (Dover Publications, New York, 2014).

[39] A. S. Davydov, Quantum Mechanics (Pergamon Press, Oxford, 1976).

[40] R. G. Newton, Scattering Theory of Waves and Particles (Springer-Verlag, New York, 1982).

[41] C. Ramsauer, Über den Wirkungsquerschnitt der Gasmoleküle gegenüber langsamen Elektronen, Ann. Phys. (Berlin) 369, 513 (1921).

[42] J. S. Townsend and V. A. Bailey, The motion of electrons in gases, Philos. Mag. 42, 873 (1921).

[43] P. G. Burke, R-Matrix Theory of Atomic Collisions (Springer-Verlag, New York, 2011). 